\title{
Potentialité des associations symbiotiques plantes-micro-organismes pour réhabiliter des sites fortement dégradés en milieu méditerranéen
}

Brigitte Brunel ${ }^{1}$

Odile Domergue $^{1}$

Lucette Maure $^{1}$

Patrice Brahic ${ }^{2}$

Antoine Galiana

Ramon $\mathrm{Josa}^{3}$

Philippe De Lajudie ${ }^{1}$

Thérèse Attallah ${ }^{4}$

Hélène Risk ${ }^{4}$

Samih El-Haij ${ }^{4}$

Jean-Claude Cleyet-Marel ${ }^{1}$

${ }^{1}$ Laboratoire des symbioses tropicales et méditerranéennes (LSTM).

Unité mixte de recherche (UMR) 113 ,

TA A-82/J,

Campus international de Baillarguet,

BP 5035,

34398 Montpellier cedex 5

<brune|@supagro.inra.fr>

$<$ odile.domergue@supagro.inra.fr>

<maure@supagro.inra.fr>

$<$ galiana@cirad.fr>

$<$ p_de.lajudie@mpl.ird.fr>

$<$ cleyet@supagro.inra.fr>

2 Direction départementale de l'agriculture et de la forêt (DDAF)

Pépinière forestière de l'État,

90 , chemin de la Pioline,

13546 Aix-en-Provence cedex 4

France

<patrice.brahic@agriculture.gouv.fr>

${ }^{3}$ Departament d'Enginyeria Agroalimentaria Biotecnol (DEAB),

Universitat politècnica de Catalunya (UPC), Edifici D4,

Avd. Canal,

Campus del Baix Llobregat,

Avinguda Del Canal Olímpic s/n

08860 Casteldefels

Espagne

<ramon.josa@upc.edu>

${ }^{4}$ Département de production végétale,

Faculté d'agronomie,

Université libanaise,

PO Box 13-5368

Beyrouth

Liban

$<$ theresea@|ynx.net.lb>

$<$ ritz@lynx.net.lb>

<elhajj@|ynx.net.lb>

Tirés à part : B. Brunel

\begin{abstract}
Résumé
Les symbioses végétales sont une composante fondamentale de la stabilité et de la durabilité des écosystèmes. L'introduction de légumineuses associées à leurs auxiliaires microbiens fixateurs d'azote ou améliorant la biodisponibilité d'éléments nutritifs, dans des sites dégradés fortement appauvris en éléments nutritifs, est une condition primordiale pour réussir l'installation de plants en milieu particulièrement contraignant, comme le sont les sites de carrière de calcaire après exploitation sous climat méditerranéen. Une stratégie de végétalisation basée sur l'ingénierie microbiologique de plants adaptés aux conditions locales, a été élaborée puis testée sur le terrain en grandeur nature dans le cas particulier de carrières de granulats calcaires. Le choix des plants symbiotiques utilisables pour la végétalisation repose, d'une part, sur la prospection d'espèces de légumineuses repérées sur des sites assez dégradés en milieu méditerranéen, faciles à multiplier et, d'autre part, sur l'identification et la production de rhizobiums partenaires efficaces. Ces plants produits en pépinière puis transférés sur le terrain présentent après trois années une bonne capacité de croissance. Les espèces arbustives sont plus particulièrement performantes avec une capacité spontanée à s'associer aux mycorhizes et à favoriser l'enrichissement en matière organique de la surface du substrat.
\end{abstract}

Mots clés : légumineuse ; pépinière ; réhabilitation des sols ; région méditerranéenne ; Rhizobium; symbiose.

Thèmes : productions végétales ; sols ; agronomie.

\section{Abstract \\ Potential of nitrogen-fixing symbiotic systems for revegetation strategies of degraded land sites in Mediterranean conditions}

Plant symbioses are fundamental for ecosystem stability and sustainability. Introduction of legumes associated with microorganisms efficient in nitrogen fixation and promoting nutrient biodisponibility in limited-nutrient degraded sites is a key step for a successful plant establishment in stress situations such as those encountered in Mediterranean soils. A vegetation strategy based on microbial engineering of locally-adapted plants was investigated and tested in field conditions within the framework of calcareous quarry rehabilitation. Choice of symbiotic plants requires, first, a prospection of native legume species growing in relatively degraded sites in Mediterranean areas and easy to multiply, and second, an identification and production of efficient rhizobial partners. Plants produced in nursery and then transplanted into the quarry showed a good capacity for establishment after three years. Shrub species performed best with spontaneous mycorrhizal association and ability to enrich topsoil with organic matter.

Key words: legumes; mediterranean region; plant nurseries; Rhizobium; soil rehabilitation; symbiosis.

Subjects: vegetal productions; soil; agronomy. 
es écosystèmes naturels subissent de fortes contraintes lorsqu'ils sont soumis au surpâturage, au passage accidentel d'incendies ou lorsqu'ils sont le théâtre d'activités industrielles comme l'ouverture de carrières d'extraction de granulats, de mines ou de nouvelles voies de communication. La pression est accentuée sur l'ensemble de l'arc méditerranéen où l'accroissement démographique régulier implique de nombreux aménagements pour satisfaire les besoins des populations en matière d'habitat et de réseaux de communication.

Les dommages liés à toutes ces perturbations sont particulièrement marqués en milieu méditerranéen où les conditions climatiques et édaphiques rendent difficile une restauration rapide des écosystèmes. Le milieu méditerranéen se caractérise par un régime de pluies particulier avec de fortes précipitations concentrées sur quelques mois de l'année, une période estivale marquée par la sécheresse et de fortes chaleurs, le tout conjugué très souvent à des sols qui se sont développés sur une roche mère calcaire. Cette dernière est à l'origine d'une contrainte supplémentaire avec une biodisponibilité souvent restreinte pour de nombreux éléments minéraux, comme en particulier le fer et le phosphore (Hinsinger, 2001).

En absence d'intervention, la végétation est dans l'incapacité de se régénérer dans les milieux détériorés et un début de cicatrisation des carrières va prendre plusieurs décennies pour les plus rapides alors que celle des déblais des anciens sites miniers peut s'échelonner sur plusieurs siècles. Des actions de réhabilitation basées sur la végétalisation peuvent être entreprises pour reconstruire un écosystème afin de réduire les impacts de l'action de l'homme dans le paysage et de limiter les risques d'érosion (Leroux, 2002). Toutefois, l'emploi de stratégies lourdes avec apport de terre de découverte ou d'amendements organiques pour tenter de réparer les dommages, outre leur coût, ne donne pas toujours les résultats escomptés, en particulier en milieu méditerranéen.

Dans ce contexte, la recherche de végétaux adaptés aux contraintes précédemment mentionnées, la multiplication de ces végétaux et leur réintroduction dans les milieux dégradés sont devenues des étapes incontournables. Celles-ci doivent tenir compte des connaissances disponibles en écologie, en physiologie végétale, en microbiologie du sol, en agronomie et impliquer des organismes techniques performants aptes à produire les végétaux capables de s'installer et de se développer dans des milieux à forte contrainte. Du point de vue biologique, les associations mutualistes entre plantes et micro-organismes du sol sont des éléments importants à prendre en compte car elles sont essentielles pour la survie, la croissance et la santé des plantes dans les écosystèmes terrestres naturels et les systèmes cultivés qui doivent être économes en intrants et dont des fonctions microbiennes du sol doivent être mieux exploitées (Brunel, 2006). Parmi les associations bénéfiques, la symbiose légumineuse-rhizobium est connue pour être une des plus répandues et des plus efficaces pour faire entrer par voie biologique de l'azote $(\mathrm{N})$ sous forme organique dans les écosystèmes. Après l'eau, l'azote étant l'un des nutriments les plus limitants de la croissance végétale, les légumineuses sont avantagées pour coloniser les milieux dégradés et appauvris en matière organique et en azote. À ce titre, la fixation biologique d'azote a un rôle clé dans la réhabilitation de sites dont l'horizon de surface a disparu suite à diverses activités humaines. Ainsi les stratégies de végétalisation incluant des plantes inoculées avec leurs micro-organismes mutualistes sont-elles les plus performantes (Requena et al., 2001).

\section{Contexte}

\section{de l'étude appliquée} au cas des carrières

\section{de calcaire}

Nous illustrerons notre démarche avec l'exemple de carrières à granulats qui constitue une situation extrême de détérioration d'écosystèmes dans le sens où la terre de surface (appelée terre végétale) ainsi que les végétaux qui s'y développent sont totalement éliminés, puis des terrasses sont creusées pour accéder aux matériaux situés dans les couches inférieures. Nous exposerons deux étapes nécessaires pour définir sur le terrain des actions d'interventions afin de végétaliser de manière durable ces sites: i) une étude menée au Liban conduisant à identifier les plants symbiotiques les plus adaptés au milieu qui est à réhabiliter ii) une action d'intervention grandeur nature dans le sud de la France avec introduction de végétaux symbiotiques produits aujourd'hui en pépinière après plusieurs années de mises au point rigoureuses. Ces végétaux, issus au départ de prospection dans le sud de la France, sont transférés sur le site à réhabiliter, puis le devenir de leur installation est diagnostiqué en suivant sur les premières années leur capacité à se développer et à initier la régénération d'un sol permettant ainsi de promouvoir une dynamique d'établissement d'un écosystème plus complexe. Quand les couples mutualistes légumineuses-symbiotes auront été choisis pour les sites particuliers à végétaliser, les technologies et la démarche développées en France seront potentiellement transférables au Liban ainsi qu'à d'autres régions méditerranéennes soumises à fortes contraintes.

\section{Exploitations de carrières et législation}

Les exploitations de carrières à granulats, de cailloux et de pierres sont indispensables pour le développement économique d'infrastructures (bâtiments, voies de communication) et d'autres produits industriels (sidérurgie, ciments, cosmétiques...). Après l'eau et l'air, il s'agit de la troisième ressource la plus consommée par notre société. La moyenne nationale française et libanaise avoisine les 6-7 tonnes par an et par habitant. La demande est plus particulièrement accentuée au nord du Bassin méditerranéen où la population se concentre autour du littoral : par exemple, la consommation par habitant s'élève à 8 tonnes en Catalogne (Espagne) (Roca Riera, 2004) et à 9 tonnes dans l'Hérault (France). Au Liban, le nombre des carrières est relativement élevé suite à la reconstruction qui a suivi la guerre de 1975-1990. Leur nombre est estimé à 710 , soit l'équivalent d'une carrière par $15 \mathrm{~km}^{2}$ (Lebanese Center for Policy Studies, 1996). C'est une activité très lucrative destinée surtout au marché local de construction (routes, immeubles...).

En France, depuis 1994 (application de la loi relative aux carrières de janvier $1993^{1}$ ), l'exploitation des carrières est soumise à la législation des Installations

\footnotetext{
${ }^{1}$ Loi n 93-3 du 4 janvier 1993 relative aux carrières. Journal officiel de la République française, 5 janvier 1993, $\mathrm{n}^{\circ} 3$. www.admi.net/ jo/HAUX9210596L.html
} 
classées pour la protection de l'environnement et comporte une obligation de remise en état (c'est-à-dire nettoyage du site et mise en sécurité) après travaux. Elle incite les sociétés à réaliser un aménagement coordonné et à végétaliser le site avant la fin de l'exploitation. Au Liban, une législation comparable (décret 8803 du 4 octobre 2002) existe mais reste moins suivie avec notamment la création de falaises dont la hauteur dépasse les $20 \mathrm{~m}$ autorisés. Cette loi a été renforcée par une décision du conseil des ministres (décision $2,77^{\mathrm{e}}$ session) qui limite la localisation des carrières à la chaîne de l'AntiLiban uniquement et oblige la réhabilitation des sites exploités par la création de terrasses et la mise en œuvre de méthodes de revégétalisation (Lebanese National Action Program, 2003).

D'une façon générale, et avant la fermeture de toute exploitation de carrière, une réhabilitation est obligatoire et celle-ci comprend nécessairement la mise en place d'une couverture végétale sur tout ou partie de l'installation. Les principaux problèmes auxquels sont confrontés les paysagistes et aménageurs de sites d'exploitation de granulats en milieu méditerranéen sont l'extrême pauvreté du milieu en éléments nutritifs, en particulier en $\mathrm{N}$ total qui n'excède jamais $0,5 \mathrm{~g} / \mathrm{kg}$, de fortes carences en anhydride phosphorique, une capacité d'échange en cations très faible et des contraintes climatiques drastiques. Les travaux lourds de génie civil avec l'utilisation de terre de découverte ou l'apport de terre végétale, outre leurs coûts, présentent de nombreux inconvénients. La terre de découverte a souvent perdu une partie de ses propriétés biologiques lors de son stockage et l'apport de terre végétale s'accompagne généralement d'une prolifération de végétaux exogènes indésirables issus des graines présentes dans le matériau rapporté. Dans ce contexte, l'utilisation de végétaux associés à des micro-organismes capables de leur conférer une réelle aptitude à se développer sur des substrats pauvres en $\mathrm{N}$ et en phosphore revêt un véritable intérêt (Devaux et Moiroud, 2001). Ces végétaux sont non seulement capables de croître rapidement sur des substrats pauvres mais aussi de modifier ces derniers grâce à un apport massif de litière enrichie en $\mathrm{N}$ et autres minéraux permettant ainsi l'installation de nouvelles espèces. Les espèces capables de se développer de façon spectaculaire sur des substrats pauvres ont la particularité de s'associer avec des bactéries fixatrices d'azote et des champignons mycorhiziens. L'enjeu est d'identifier pour les différentes zones climatiques et les différents substrats, les meilleures associations plantes-micro-organismes, puis de les réassocier en pépinière pour les transférer dans les milieux dégradés où elles exprimeront leur potentiel.

\section{Choix du matériel biologique}

\section{Prospection des espèces végétales adaptées localement}

Au niveau du Liban, le choix s'est porté sur une région au nord de la capitale dans laquelle trois étages de végétation de l'ensemble méditerranéen sont représentés: thermoméditerranéen $\quad(<500 \mathrm{~m}$ d'altitude), mésoméditerranéen (500$1000 \mathrm{~m}$ ) et supraméditerranéen (1000$1600 \mathrm{~m})$. Une quinzaine de sites a été sélectionnée (176-1302 m) dont le couvert végétal variait entre les pâturages naturels/zones herbacées et les zones arbustives/forêts dégénérées et dont l'origine de la dégradation était le surpâturage, l'urbanisation ou leur proximité avec des carrières. Les sites prospectés en Francesont tous situés dans l'étage thermoméditerranéen.

Le niveau de dégradation a été estimé entre mars et mai sur la base du recouvrement au sol des plantes, du nombre d'espèces et d'individus. On a distingué trois sites à dégradation élevée (recouvrement au sol $<40 \%$ ), 7 à niveau moyen (recouvrement entre 40-70\%) et 5 à niveau bas. À mesure que la dégradation s'accentuait, le nombre d'espèces et d'individus diminuait.

Quel que soit le niveau de dégradation, les légumineuses étaient bien représentées en nombre d'espèces et d'individus. L'abondance des espèces a été évaluée selon une échelle de 1 à 5 . Seize espèces étaient très abondantes ( $>5$ sites), 9 abondantes ( $4-5$ sites), 2 moyennement (3-4 sites), 8 rares ( 2 sites) et 17 très rares (1 site). La distribution des espèces de légumineuses selon le niveau de dégradation a aussi été étudiée. Ainsi, 10 espèces $(18,5 \%)$ se trouvaient à tous les niveaux de dégradation, 24 espèces (44,4\%) dans des sites moyennement dégradés, 10 espèces dans des sites peu dégradés et 10 espèces dans des sites fortement dégradés. Une quinzaine d'espèces, appartenant aux genres Calicotome, Medicago, Physanthyllis, Psoralea, Securigera, Spartium, Trifolium et Vicia ont ainsi été considérées comme potentiellement utiles.

Lors d'une autre approche consistant à étudier la végétation spontanée de carrières calcaires, Khater (2004) a trouvé la famille des Légumineuses bien représentée (11,8\% des espèces) après celle des Astéracées (14,8\%). Le choix définitif des espèces utilisables pour la végétalisation devra prendre en compte, outre leur caractère arbustif ou herbacé, la facilité de multiplication de façon à disposer de semences et de plants en quantités suffisantes.

\section{Choix des partenaires symbiotiques microbiens}

Pour choisir les partenaires bactériens des espèces de légumineuses au Liban, la nodulation de l'hôte a d'abord été vérifiée. La plupart des espèces herbacées étaient nodulées in situ (El-Alia, 2002). Dans le cas de plantes ligneuses ou même pérennes herbacées, l'observation des nodosités au niveau des jeunes racines est peu aisée. Dans certains cas, il a fallu recourir au piégeage par la plante (Calicotome, Psoralea, Dorycnium, Genista) pour obtenir un nombre satisfaisant de nodosités avant d'isoler les souches de rhizobium. Leur caractérisation moléculaire a été entreprise (Zakhia, 2004). Les souches de rhizobium utilisées en France pour l'inoculation des légumineuses et leur production en pépinière ont fait l'objet d'une sélection en serre sur leur capacité à fixer l'azote atmosphérique.

\section{Optimisation}

\section{de la production de plants symbiotiques en pépinière}

Un travail important a été consacré, en France, à la production expérimentale en pépinière, de plants de légumineuses en les associant de façon efficace avec leurs bactéries fixatrices d'azote spécifiques afin d'optimiser le potentiel de survie et de croissance de ces végétaux après 
transfert dans des milieux dégradés. Toutes les semences ont été préalablement scarifiées avant le semis afin de lever la dormance tégumentaire, puis transférées après germination sur un substrat de culture placé dans des containers spéciaux pour éviter le développement des racines en " chignon". Le substrat de culture était constitué d'un mélange de $50 \%$ d'écorce de pin compostée et de $50 \%$ de tourbe additionné de carbonate de calcium afin d'obtenir un $\mathrm{pH} \geq 7$. Les plants ont été inoculés avec la souche de rhizobium adéquate. Les plants ont été alimentés de juin à novembre avec une solution nutritive (N-P-K : 4-6-8) diluée à $5 \%$ à raison de 10 litres par $\mathrm{m}^{2}$ et par semaine. Plusieurs volumes de containers ont été testés et, finalement, nous avons retenu un volume de $400 \mathrm{~mL}$. Le volume de substrat et la forme du container autorisent ainsi un développement optimal en pépinière d'un système racinaire portant de nombreuses nodosités et permettent aux racines de croitre rapidement en profondeur et de bien valoriser la réserve hydrique du substrat.

\section{Plantation et suivi des plants introduits}

Les plants de légumineuses produits en pépinière ont été transférés sur le terrain dans le substrat de la carrière au printemps 2001 (figure 1). Le substrat qui sert de support aux plants est un matériel très caillouteux ne contenant que $17 \%$ de terre fine (diamètre des particules $<2 \mathrm{~mm}$ ). Il s'agit d'un support reconstitué à partir des stériles de la carrière (partie des matériaux non commercialisables), et de quelques déblais organiques et minéraux mélangés, n'ayant pas encore subi l'action de pédogenèse (ces déblais sont qualifiés d'" anthropogéomorphiques spoliques " selon l'Organisation des Nations unies pour l'agriculture et l'alimentation (FAO, 1998). Son $\mathrm{pH}$ est de 8,3 avec une faible teneur en $\mathrm{N}(0,21 \mathrm{~g} \mathrm{~N} / \mathrm{kg}$ de sol), en $\mathrm{P}\left(0,020 \mathrm{~g} \mathrm{P}_{2} \mathrm{O}_{5} / \mathrm{kg}\right.$ de sol) et en $\mathrm{C}$ organique $(2 \mathrm{~g} \mathrm{C} / \mathrm{kg}$ de $\mathrm{sol})$.

\section{Estimation de la biomasse végétale sur les premières années après plantation}

Les parties aériennes des plants introduits ont été mesurées sur une période de

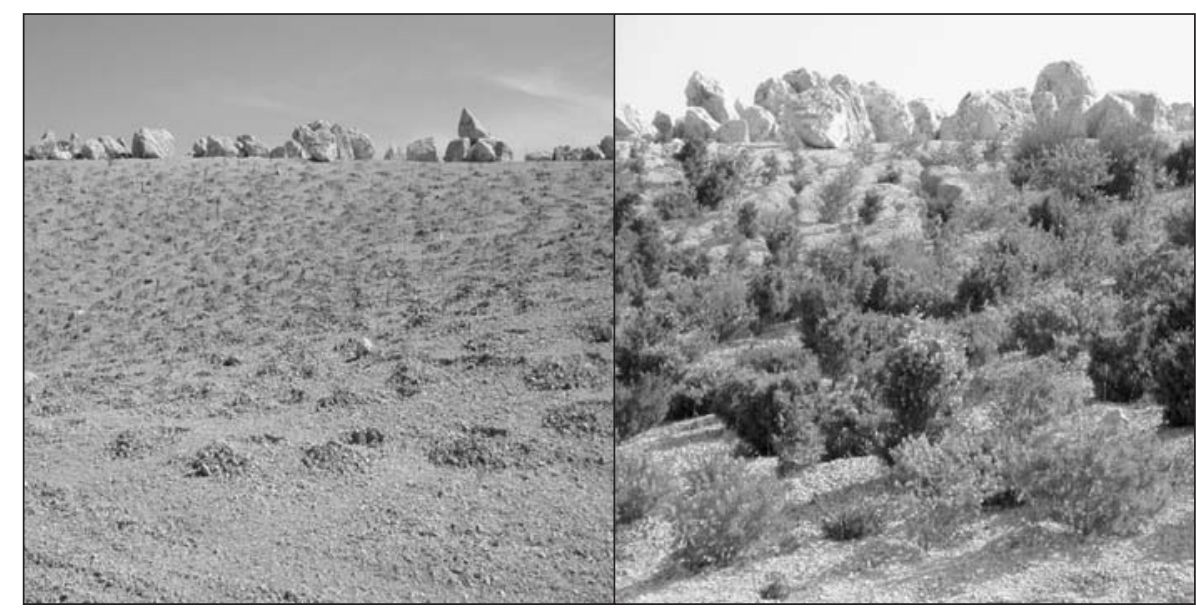

Figure 1. Aperçu d'un site expérimental de réhabilitation d'une carrière. (Société J. Lefèvbre, Châteauneuf-lès-Martigues, France).

Figure 1. View of the rehabilitation trial site (quarry of Company J. Lefèvbre, Châteauneuf-lès-Martigues, France).

Au moment de la plantation (à gauche) et après 3 années (à droite).
3 ans après plantation (figure 1). Les plus forts taux de croissance ont été obtenus pour quatre légumineuses arbustives: Coronilla glauca, C. varia, Medicago arborea et Dorycnium birsutum (figure 2). Alors que $M$. arborea s'installe correctement sur plus de 4 ans sur des sites du Sud-Est de la France, cette plante associée à son cortège symbiotique s'est avérée peu performante dans le Sud-Est de l'Espagne (Herrera et al., 1992).

\section{Détection de mycorhizes}

Les plants introduits n'avaient pas été inoculés avec leurs partenaires mycorhiziens en pépinière. Après 3 années de culture dans le substrat de la carrière, tous les plants sont naturellement colonisés par des mycorhizes à arbuscules (figure 3) qui constituent la structure fonctionnelle d'échange avec la plante. Certaines espèces végétales sont forte-

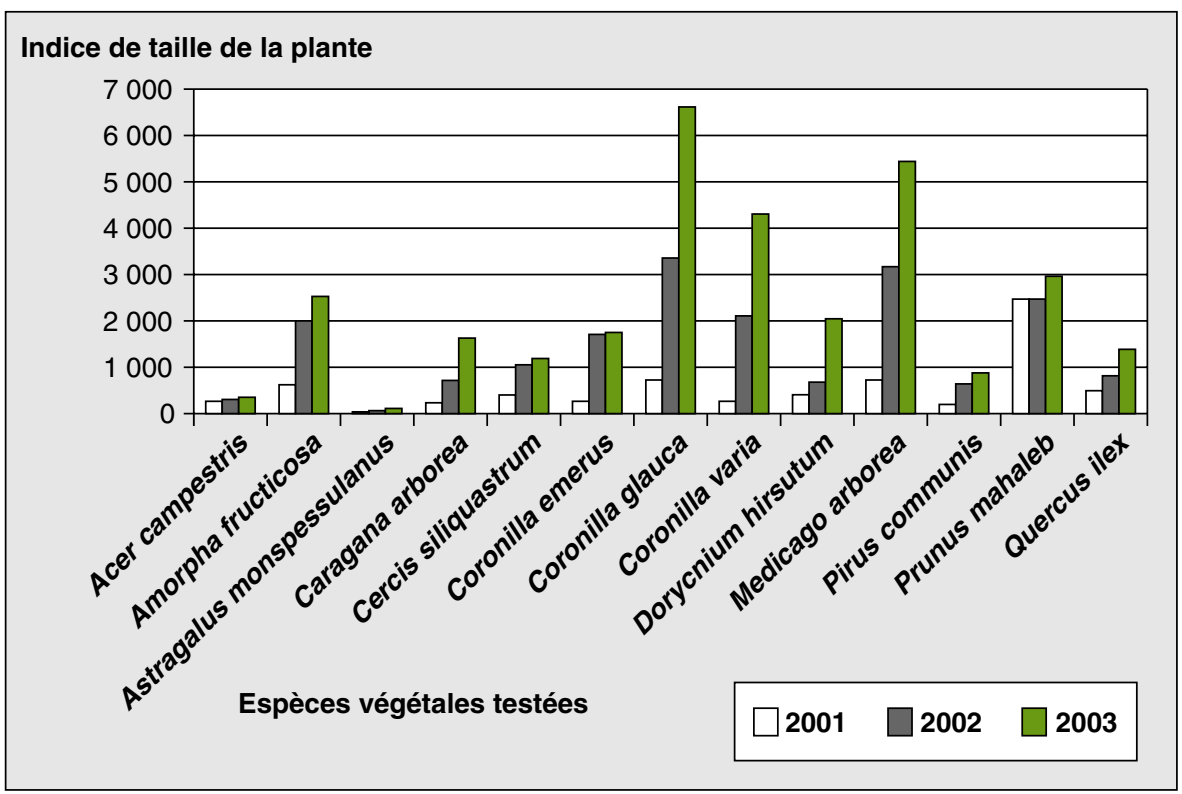

Figure 2. Suivi de la production de biomasse végétale aérienne sur 3 ans après plantation.

Figure 2. Above-ground plant biomass production over 3 years after transplantation. Indice de taille de la plante $=$ hauteur $\mathrm{x}$ diamètre des parties aériennes $\left(\mathrm{cm}^{3}\right)$. 


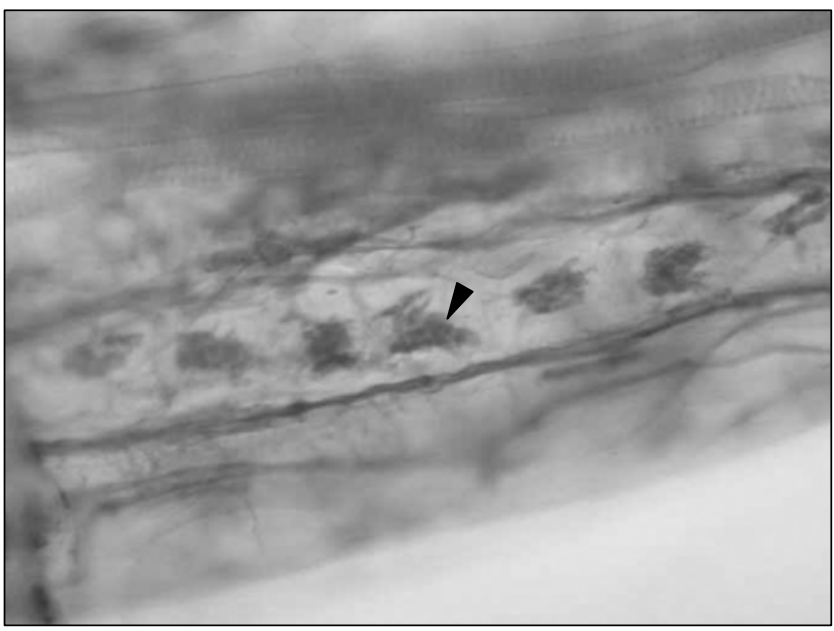

Figure 3. Détection de mycorhizes à arbuscules dans les cellules racinaires de Coronilla glaucae.

Figure 3. Detection of arbuscular mycorrhizae in root cells of Coronilla glaucae.

La flèche indique une arbuscule après coloration au bleu Trypan. ment infectées (C. glauca, 91,0\%; M. arborea, $92,7 \%$ ) alors que d'autres espèces comme C.varia $(75,7 \%)$ ou Tussilago farfara (59,0\%), plantes se développant spontanément à proximité, sont moins infectées. Ces niveaux de colonisation par des mycorhizes natives s'expliquent sans doute par leurs propriétés d'ubiquité et de spécificité à large spectre vis-à-vis de leur hôte végétal contrairement aux rhizobiums qui sont spécifiques.

\section{Teneur \\ en matière organique et valeur du rapport $\mathrm{C} / \mathrm{N}$ en surface}

Du point de vue de la restauration du substrat du sol, un horizon organique de surface mince $(0-5 \mathrm{~mm})$ et discontinu s'est formé par accumulation de litière après 3 années de végétalisation. La teneur en $\mathrm{C}$ organique est élevée et hétérogène (119 g C/kg de sol \pm 130$)$ sous les plants arbustifs de C. glauca, $M$. arborea et $D$. hirsutum (figure 4). Dans les 5 premiers centimètres du matériel spolique végétalisé, de l'azote total a été légèrement incorporé $(0,3$ à $1,0 \mathrm{~g} \mathrm{~N} / \mathrm{kg}$ de sol) en comparaison avec le matériel non végétalisé. Une légère augmentation de la biomasse microbienne a été mesurée dans le sol rhizosphérique de C. glauca (de 1 à 28 mg C/kg de sol).

L'introduction de légumineuses dans les stériles de restauration favorise l'enrichissement du sol en matière organique. Cet enrichissement se forme sous la protection directe de chaque plant végétal et son épaisseur est liée à sa proximité avec le pied de la plante. Le plus fort taux de matière organique apparaît sous les plants de D. hirsutum avec jusqu'à $120 \mathrm{~g}$ $\mathrm{C} / \mathrm{kg}$ de terre fine (figure 4). L'architecture de cette plante de type couvre-sol est favorable à la rétention de résidus végétaux après leur chute. Cet enrichissement est décelable aussi dans les 5 centimètres supérieurs du substrat et dans les mêmes circonstances.
Les rapports $\mathrm{C} / \mathrm{N}$ mesurés dans les échantillons de substrat varient de 32 à 13, indiquant des conditions favorables pour la bioactivation du milieu grâce à la minéralisation microbienne. Les meilleures conditions se trouvent plutôt dans l'horizon organique (rapport $\mathrm{C} / \mathrm{N}$ entre 13 et 16), bien que les valeurs trouvées dans les 5 premiers centimètres du substrat indiquent aussi une teneur en $\mathrm{N}$ suffisante pour soutenir une activité microbienne substantielle (18 à 32).

\section{Conclusion}

Nous proposons une stratégie pour végétaliser les écosystèmes dégradés en valorisant les propriétés biologiques des associations plantes-micro-organismes. L'utilisation de légumineuses indigènes du pourtour méditerranéen et l'inoculation en pépinière avec des bactéries fixatrices d'azote sélectionnées sont les premiers éléments du succès de l'installation rapide de ces végétaux dans les milieux fortement carencés en azote. Le troisième élément du succès est l'utilisation d'un substrat et d'un container compatibles avec un développement optimal du système racinaire et la formation de nombreuses nodosités fixatrices d'azote.

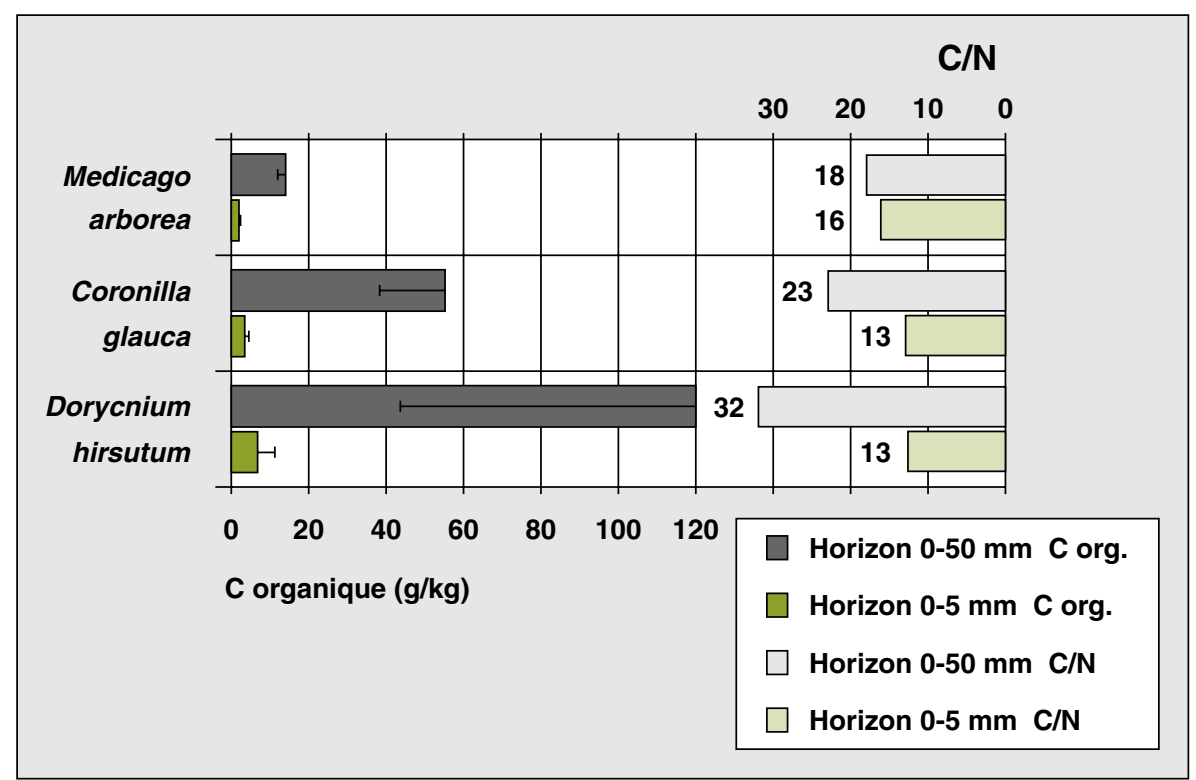

Figure 4. Teneur en carbone organique (à gauche) et valeur du rapport $\mathrm{C} / \mathrm{N}$ de la matière organique (à droite) du sol rhizosphérique associé à 3 légumineuses.

Figure 4. Carbon organic matter (left) and $\mathrm{C} / \mathrm{N}$ ratio of organic matter (right) from rhizospheric soil associated with 3 legumes.

Les barres représentent les écarts types $(n=3)$. 
L'assemblage de ces trois éléments conduit à la constitution d'un système biologique plantes-micro-organismes qui agit comme un catalyseur favorisant la survie et le développement des végétaux après leur transfert dans les conditions fortement contraignantes caractérisées par une saison sèche et chaude, et un milieu abiotique de roches calcaires plus ou moins divisées. L'accumulation progressive d'une litière riche en azote est de nature à favoriser l'installation d'une flore plus riche et diversifiée qui favorisera l'insertion paysagère des anciens sites d'exploitation de granulats.

\section{Remerciements}

Les auteurs remercient les sociétés Jean Lefèvbre Méditerranée (carrière de Châteauneuf) et GSM (France) pour la mise à disposition de sites expérimentaux, et le programme de coopération francolibanais CEDRE pour leur aide financière.

\section{Références}

Lebanese Center for Policy studies (LCPS) Lebanon Report 1996 ; (3) : 26 p.

www.unccd.int/cop/reports/asia/national/2000/ lebanon-eng.pdf.

Lebanese National Action Program, Ministry of Agriculture. Report 2003. Beyrouth : Ministry of Agriculture, 2003.

Brunel B. La microbiologie des sols: un champ prometteur pour l'agro-écologie. In Pour une gestion intégrée et durable de la
production agricole en France. Paris: Club production agricd

Devaux F, Moiroud A. Un atout pour revégétaliser et revivifier les terrains dégradés. Arbo rescences $2001 ; 89: 20-3$.

El-Alia R. Spontaneous nodulation of native wild legumes in Lebanon. Graduation project Faculty of Agricultural Sciences, Lebanese University, 2002.

Organisation des Nations unies pour I'agriculture et I'alimentation (FAO) ; International Soil Reference and Information Centre (ISRIC) International Society of Soil Science (ISSS). World reference base for soil resources. Report 84. Rome : FAO, 1998.
Herrera MA, Salamanca CP, Barea JM. Inoculation of woody legumes with selected arbus cular mycorrhizal fungi and rhizobia to recover desertified mediterranean ecosystems. Appl Environ Microbiol 1992 ; 59 : 129-33.

Hinsinger P. Bioavailability of soil inorganic $P$ in the rhizosphere as affected by root-induced chemical changes : a review. Plant Soil 2001 . 237 : 173-95.

Khater C. Dynamiques végétales postperturbations sur les carrières calcaires au Liban. Stratégies pour l'écologie de la restauration en régions Méditerranéennes. Thèse de doctorat, université de Montpellier II, 2004.

Leroux $C$. La réhabilitation des mines et carrières à ciel ouvert. Bois For Trop 2002; 272 : 5-19.

Requena N, Perez-Solis E, Azcón-Aguilar C Jeffries P, Barea JM. Management of indigenous plant-microbe symbioses aids restoration of desertified ecosystems. Appl Environ Microbiol $2001 ; 67$ : 495-8.

Roca Riera P. Compareixença dels representants del Gremi d'Arids de Catalunya perquè expliquin la previsió que hi ha en l'abastament d'àrids per a l'obra pública a Catalunya fins al 2007. Diari de sessions del Parlament de Catalunya, 1 desembre de 2004, Sèr C 2004; 121 : 3-16.

Zakhia F. Diversité des bactéries hôtes de légumineuses Méditerranéennes en Tunisie et au Liban. Thèse de doctorat, université de Montpellier II, 2004. 